\title{
Induced Refolding of a Temperature Denatured Llama Heavy-Chain Antibody Fragment by Its Antigen
}

\author{
Edward Dolk, ${ }^{1}$ Cees van Vliet, ${ }^{2}$ Janice M.J. Perez, ${ }^{2}$ Gert Vriend, ${ }^{4}$ Hervé Darbon, ${ }^{3}$ Gilles Ferrat, ${ }^{3}$ \\ Christian Cambillau, ${ }^{3}$ Leon G.J. Frenken, ${ }^{2}$ and Theo Verrips ${ }^{1,2 *}$ \\ ${ }^{1}$ Department of Molecular and Cellular Biology, University of Utrecht, Utrecht, The Netherlands \\ ${ }^{2}$ Unilever Research Vlaardingen, Olivier van Noortlaan 120, Vlaardingen, The Netherlands \\ ${ }^{3}$ Architecture et Fonction des Macromolécules Biologiques, CNRS, UMR 6098, Marseille Cedex, France \\ ${ }^{4}$ CMBI, KUN, Toernooiveld 1, Nijmegen, The Netherlands
}

\begin{abstract}
In a previous study we have shown that llama VHH antibody fragments are able to bind their antigen after a heat shock of $90^{\circ} \mathrm{C}$, in contrast to the murine monoclonal antibodies. However, the molecular mechanism by which antibody:antigen interaction occurs under these extreme conditions remains unclear. To examine in more detail the structural and thermodynamic aspects of the binding mechanism, an extensive CD, ITC, and NMR study was initiated. In this study the interaction between the llama VHH -R2 fragment and its antigen, the dye Reactive Red-6 (RR6) has been explored. The data show clearly that most of the VHH-R2 population at $80^{\circ} \mathrm{C}$ is in an unfolded conformation. In contrast, $C D$ spectra representing the complex between VHH-R2 and the dye remained the same up to $80^{\circ} \mathrm{C}$. Interestingly, addition of the dye to the denatured VHH-R2 at $80^{\circ} \mathrm{C}$ yielded the spectrum of the native complex. These results suggest an induced refolding of denatured VHH-R2 by its antigen under these extreme conditions. This induced refolding showed some similarities with the well established "induced fit" mechanism of antibodyantigen interactions at ambient temperature. However, the main difference with the "induced fit" mechanism is that at the start of the addition of the antigen most of the VHH molecules are in an unfolded conformation. The refolding capability under these extreme conditions and the stable complex formation make VHHs useful in a wide variety of applications. Proteins 2005;59:555-564.

$\odot 2005$ Wiley-Liss, Inc.
\end{abstract}

Key words: folding; NMR; ITC; CD spectroscopy; VHH; camelid; denaturation; stability

\section{INTRODUCTION}

Antibody-antigen interaction has been well described in recent years. On complex formation, conformational changes of antibody and/or antigen can occur. Koshland ${ }^{1}$ first described conformational changes that induced an active state in an enzyme, subtilisin, by a substrate molecule; this mechanism was called "induced fit." This mechanism can be described as fast bimolecular association followed by a unimolecular isomerization. Meanwhile, examples of this "induced fit" mechanism have been de- scribed for both antibody-antigen interaction as well as other protein-protein interactions and protein-DNA interactions. $^{2-4}$

The equilibrium between the complex and the uncomplexed antigen and antibody fragment is described by a simple model, the law of mass action. The equilibrium will set when the rate at which new antibody-antigen complexes are formed equals the rate at which the antibodyantigen complexes dissociate. However, whether the equilibrium is directed towards the complex or the dissociated antibody and antigen depends on many factors, like the temperature. Extreme conditions necessary for a lot of applications in research or the industry can shift this equilibrium in such a way that the antibodies are not useful any more.

A novel class of $\operatorname{IgG}$ antibodies was discovered in camelids by Hamers-Casterman and coworkers. ${ }^{5}$ These antibodies are devoid of light chains and are referred to as heavy-chain antibodies. The binding part of the heavy-chain antibodies comprises only a single domain, called VHH. Camelid VHHs display similar functional characteristics with respect to specificity and affinity compared to classical antibodies. ${ }^{6,7}$ We have shown that the llama VHH antibody fragments can be produced at high levels in Saccharomyces cerevisiae, up to $2.5 \mathrm{mg}$ $\mathrm{L}^{-1} \mathrm{OD}_{660}{ }^{-1}$ in shake flask or 10 -fold higher with fed-batch fermentation at $10 \mathrm{~L}$ scale. ${ }^{8-10}$ A remarkable difference between the llama antibody fragments and the convential antibody fragments is the apparently higher heat stability of the llama VHHs. ${ }^{6}$ Van der Linden showed that some of the VHH fragments were able to bind their antigen even at a temperature as high as $90^{\circ} \mathrm{C}$. Other fragments were found not able to bind their antigens at this temperature, but could bind their

The Supplementary Materials referred to in this article can be found at http://www.interscience.wiley.com/jpages/0887-3585/suppmat

The first two authors contributed equally to the presented work.

Grant sponsor: the European Community BIOTECH Structural Biology; grant number: BIO4 CT98-048.

*Correspondence to: Theo Verrips, Department of Molecular and Cellular Biology, University of Utrecht, Padualaan 8, $3584 \mathrm{CH}$ Utrecht, The Netherlands.E-mail: c.t.verrips@bio.uu.nl

Received 19 January 2004; Accepted 24 September 2004

Published online 18 March 2005 in Wiley InterScience (www.interscience.wiley.com). DOI: 10.1002/prot.20378 
antigen upon cooling down. We have shown that one of these llama VHH antibody fragments against the human pregnancy hormone hCG is indeed able to refold into its native conformation after exposure to high temperatures. ${ }^{11}$ The question arose as to whether the $\mathrm{VHH}$ antibody fragments that can bind their antigen at high temperatures still have their native structure or whether refolding of the antibody takes place induced by addition of the antigen.

To obtain more insight into the mechanism of binding at these extreme temperatures, we have used the $\mathrm{VHH}$ fragment R2, which binds specifically to the copper containing azo-dye RR6. ${ }^{12}$ This chemical antigen, the azo-dye RR6, has the advantage that it is not altered by the extreme conditions used in these experiments. CD spectroscopy was used to determine if the VHH-R2 fragment was completely unfolded at high temperatures and whether addition of the antigen at high temperatures could induce complex formation of the VHH-R2 fragment and its antigen. Other antibody fragments and different antigens were used to determine if this mechanism was generic or specific for VHH-R2. NMR spectroscopy was used to obtain more detailed information on the structure of VHH-R2 during unfolding, binding, and refolding. Isothermal titration calorimetry was used to investigate the thermodynamic aspects of the interaction between VHH-R2 and the dye.

We have found that the majority of the population of VHH-R2 molecules is unfolded at $80^{\circ} \mathrm{C}$. However, the preformed complex of VHH-R2 and the dye is not affected by the increase in temperature. Surprisingly, addition of the antigen at $80^{\circ} \mathrm{C}$ was able to refold $\mathrm{VHH}-\mathrm{R} 2$ to its native conformation.

The remarkable refolding properties of $\mathrm{VHH}$ domains and the stability of the complex make them very suitable for various applications. For instance, we have shown that they can be used over 2000 times in affinity purification without any measurable loss of binding capability. ${ }^{13}$ But also for applications in protein arrays they proved to be very suitable. ${ }^{14}$ On one hand, they can make very stable complexes with their antigen with binding properties exceeding traditional antibodies; on the other hand, the chemically modification necessary to link them on a support does not alter their binding properties.

\section{MATERIAL AND METHODS}

\section{Fragments Used in This Study}

The fragments used in this study are the binding domains of Llama heavy-chain antibodies (VHH). VHH-R2 against the azo-dye RR6, VHH-A52 against the azo-dye RR1, and VHH-H14 against the human pregnancy hormone hCG were used in this study. The isolation of VHHs against the azo-dye RR6, the azo-dye RR120 (dimer of RR1) and the human pregnancy hormone (hCG) has been described elsewhere. ${ }^{9,15}$ The VHHs were produced in Saccharomyces cerevisiae via batch fermentation. ${ }^{12}$ VHHs were purified using a 5-mL ProteinA column (Hi-Trap, Pharmacia). Uniformly ${ }^{15} \mathrm{~N}$-labeled VHH-R2 was pro- duced by using ${ }^{15} \mathrm{~N}$-labeled ammonium sulphate as the sole nitrogen source. The affinities of the antibodies were in the nanomolar range. ${ }^{6} \mathrm{VHH}-\mathrm{R} 2, k_{\text {on }}=1.54 \times 10^{5} \mathrm{M}^{-1}$ $\mathrm{s}^{-1} ; k_{\text {off }}=3.34 \times 10^{-3} \mathrm{~s}^{-1} ; K_{D}=22.0 \mathrm{~nm}$.

\section{Circular Dichroism}

CD spectra were collected using a thermostated (20$80^{\circ} \mathrm{C}$ ) Jobin-Yvon CD6 Dichrograph Circular Dichroïsm spectrophotometer. The data were processed with the Dichrograph software version 1.3. Experiments in the near-ultraviolet (UV) region (250-350 nm) were performed with $37 \mu \mathrm{M}$ antibody fragment in PBS pH 7.4 in $10 \mathrm{~mm}$ cylindrical quartz cuvettes (Helma). The reactive chloride groups of the azo-dye RR6 were blocked in $1 \mathrm{M}$ $\mathrm{NH}_{4} \mathrm{Cl}$. After blocking, the RR6 was transferred to PBS buffer $\mathrm{pH}$ 7.4. The spectral bandwidth was automatically kept at $2 \mathrm{~nm}$. The wavelength increment was 0.2 $\mathrm{nm} / \mathrm{step}$ and the accumulation time was $1 \mathrm{~s} / \mathrm{step}$. Each spectrum resulted from averaging five successive scans. The spectra of the solvent alone and solvent with RR6 or RR1 were recorded under identical conditions and were subtracted from the protein spectra.

Experiments in the far-UV region (190-250 nm) were performed with $90 \mu \mathrm{M}$ peptide in $\mathrm{ddH}_{2} \mathrm{O}$ in $2 \mathrm{~mm}$ quartz cuvettes (Helma). The reactive chloride groups of the azo-dye RR6 were blocked in $1 \mathrm{M} \mathrm{NH}_{4} \mathrm{Cl}$. After blocking, the RR6 was transferred to PBS buffer $\mathrm{pH}$ 7.4. Each spectrum resulted from averaging five successive scans. The spectra of the solvent alone and solvent with RR6 were recorded under identical conditions and were subtracted from the peptide spectra.

\section{NMR}

For the NMR measurements, uniformly ${ }^{15} \mathrm{~N}$-labeled VHH-R2 samples were prepared in a buffer containing 10 $\mathrm{mM}$ deuterated sodium acetate $(99.5 \% \mathrm{D}) \mathrm{pH} 4.6$, and 100 $\mathrm{mM} \mathrm{NaCl}$. The protein concentration was $0.1 \mathrm{mM}$ and the samples contained $90 \% \mathrm{H}_{2} \mathrm{O}$ and $10 \% \mathrm{D}_{2} \mathrm{O}(99.9 \% \mathrm{D})$. All NMR data sets were collected on a Bruker $600 \mathrm{MHz}$ DMX spectrometer equipped with a 5 -mm inverse tripleresonance probehead $\left({ }^{1} \mathrm{H} /{ }^{15} \mathrm{~N} /{ }^{13} \mathrm{C}\right)$ with a self-shielded z-gradient coil and a variable temperature unit. $\left[{ }^{15} \mathrm{~N},{ }^{1} \mathrm{H}\right]$ water flip-back HSQC (HSQC) spectra were measured. ${ }^{16}$ The spectral width and number of complex points in the ${ }^{15} \mathrm{~N}$ dimension was $1672 \mathrm{~Hz}$ (folding-in some backbone resonances) and 128, respectively, and $9259 \mathrm{~Hz}$ and 2048 in the ${ }^{1} \mathrm{H}$ dimension. Quadrature detection in the indirectly detected dimensions was accomplished using the States-TPPI acquisition method. ${ }^{17} \mathrm{NMR}$ data were processed using XWINNMR software from Bruker. All dimensions were apodised using shifted sine-bell windows and zero-filled to the next power of two to improve the digital resolution.

\section{Isothermal Titration Calorimetry}

ITC was performed using a Microcal Omega titration microcalorimeter (Microcal, Inc.) connected to an external waterbath. All measurements were performed using 
A.

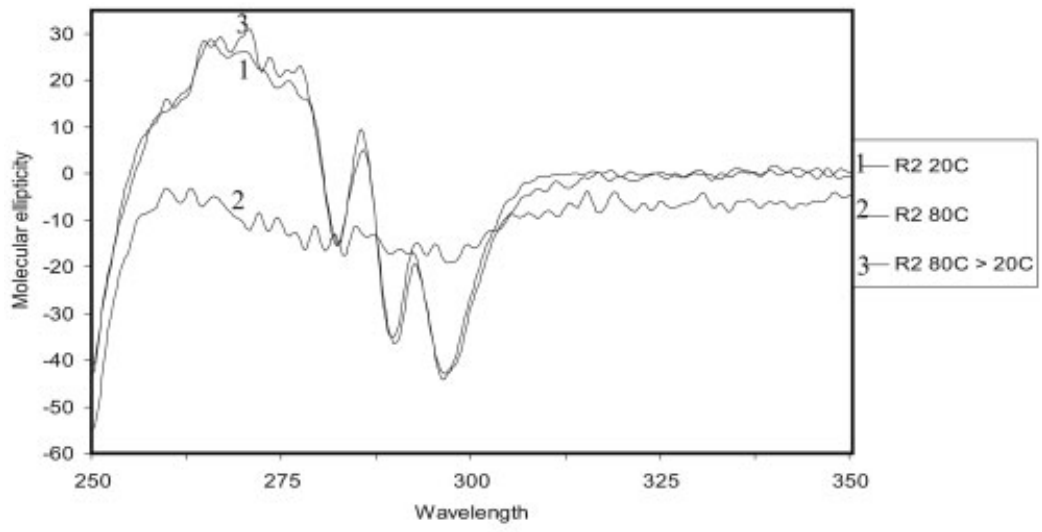

B.

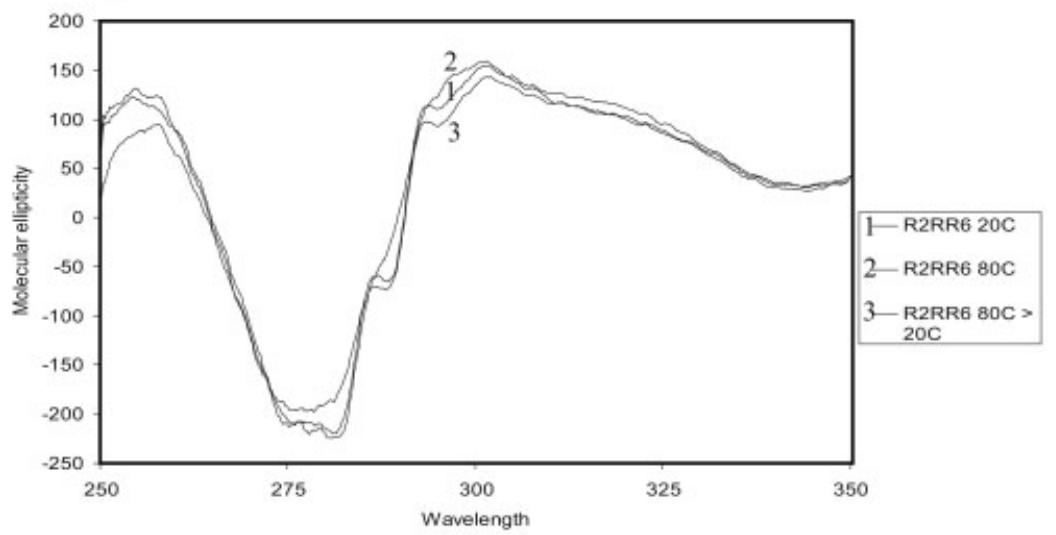

C.

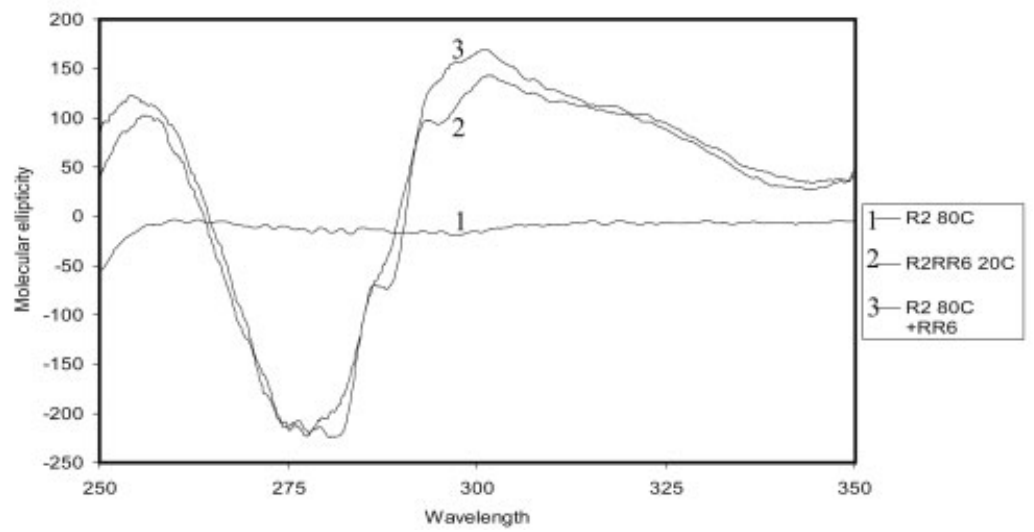

Fig. 1. (A) CD spectra in the near-UV area (250-350 nm) of $\mathrm{VHH}-\mathrm{R} 2$ at $20^{\circ} \mathrm{C}$ (line 1), $80^{\circ} \mathrm{C}$ (line 2 ), and after cooling down to $20^{\circ} \mathrm{C}$ (line 3). (B) CD spectra in the near-UV area (250-350 nm) of the VHH-R2:RR6 complex at $20^{\circ} \mathrm{C}$ (line 1), $80^{\circ} \mathrm{C}$ (line 2) and after cooling down to $20^{\circ} \mathrm{C}$ (line 3). (C) CD spectra in the near-UV area (250-350 nm) of VHH-R2 at $80^{\circ} \mathrm{C}$ (line 1), of the VHH-R2:RR6 complex at $20^{\circ} \mathrm{C}$ (line 2), and of VHH-R2 at $80^{\circ} \mathrm{C}$ after addition of a 1:1 molar ratio of preheated RR6 (line 3 ).

samples which were degassed just before use. To investigate the binding of RR6 to VHH-R2 experiments were performed with $33 \mu \mathrm{M}$ antibody fragment in $\mathrm{PBS} \mathrm{pH} 7.4$ in the cell and $0.875 \mathrm{mM}$ blocked RR6 in PBS buffer $\mathrm{pH} 7.4$ in the syringe. Cell volume was $1345 \mathrm{~mL}$ of VHH-R2. Fifty injections of $6 \mu \mathrm{L}$ of RR 6 were performed. The solution was stirred at $700 \mathrm{rpm}$. In all cases, injections were carried out over a 15-s period with a 4-min equilibration period between each injection, which was sufficient for the baseline to be reestablished. Data analysis was performed 
A.

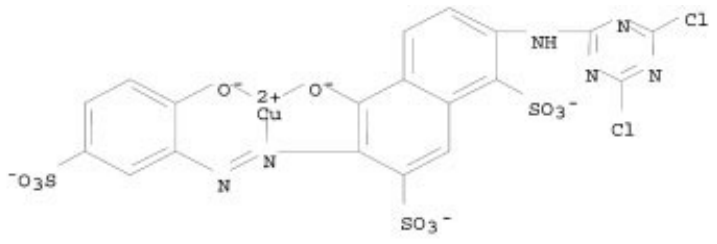

Molecular stucture of RR6

B.

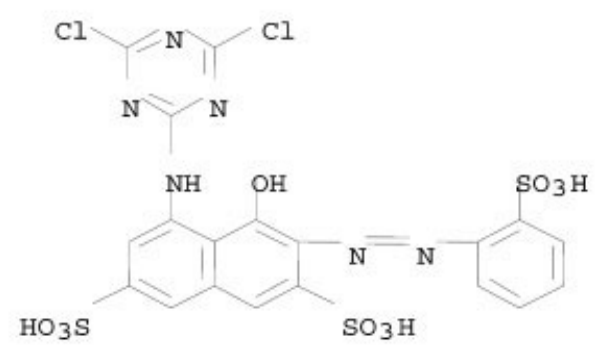

Molecular stucture of RR1

Fig. 2. Structure of the copper containing azo-dye RR6 (A) and the azo-dye RR1 (B).

using the ITC data analysis software Microcal origin (Microcal, Inc.).

\section{ELISA Affinity Measurements}

Polysorp plates were coated with $0.5 \mathrm{mM}$ blocked RR 1 or RR6 in $100 \mu \mathrm{L} /$ well at $50^{\circ} \mathrm{C}$ for $2 \mathrm{~h}$. Plates were blocked with $200 \mu \mathrm{L} 4 \%$ Marvel in PBS. A dilution range of purified VHH was incubated for $1 \mathrm{~h}$ at room temperature in $2 \%$ Marvel, $0.05 \%$ Tween-20 in PBS (100 $\mu \mathrm{L} / \mathrm{well})$. After washing with PBS/Tween $(0.05 \%)$, the plates were incubated with polyclonal rabbit-antillama IgG and goatantirabbit immunoglobulins conjugated to horseradish peroxidase (Jackson Immunoresearch Lab Inc.) in 0.05\% Tween-20/PBS, $100 \mu \mathrm{L} /$ well. Finally, the peroxidase enzyme-activity was determined with $o$-phenylenediamine (OPD) as a substrate. The optical density was measured at $490 \mathrm{~nm}$ after termination of the reaction by addition of 50 $\mu \mathrm{L} 1 \mathrm{M} \mathrm{H}_{2} \mathrm{SO}_{4}$.

\section{Spectroscopy}

\section{RESULTS AND DISCUSSION}

To collect information on folding of VHH at high temperatures, CD spectroscopy was used. Protein aggregation due to the high concentration of $\mathrm{VHH}$ at $80^{\circ} \mathrm{C}$ did not allow us to asses folding with experiments in the far-UV area (190-250 $\mathrm{nm}$ ) (see supplementary data). To investigate the tertiary structure of the $\mathrm{VHH}$ antibody fragments, $\mathrm{CD}$ spectra were taken in the near-UV area $(250-350 \mathrm{~nm})$ at different temperatures. Figure 1(A) shows the CD spectrum of VHH-R2 at $20^{\circ} \mathrm{C}$, which is considered to be the native state. The clear negative peaks shown in this spectrum at 281, 290, and 297 $\mathrm{nm}$ represent the $\mathrm{CD}$ bands of the aromatic residues present in the VHH-R2 molecule (line 1), in particular, the stacking interactions of the chromophor side chains of the tryptophane molecules. Subsequent incubation of the VHH-R2 sample at $70^{\circ} \mathrm{C}$ for 120 min does not affect the $\mathrm{CD}$ spectrum (data not shown), indicating that the structure of the fragment remains unchanged. However, incubating the VHH-R2 at $80^{\circ} \mathrm{C}$ for $10 \mathrm{~min}$ dramatically alters the $\mathrm{CD}$ spectrum of VHH-R2, resulting in the disappearance of the CD bands (line 2).

The hydrophobic stacking interactions between the chromophor side chains are disturbed, indicating an unfolded conformation of the fragment. Subsequent cooling of the VHH-R2 sample back to $20^{\circ} \mathrm{C}$ rendered the native spectrum (line 3). This indicates that heat induced unfolding of VHH-R2 is reversible, comparable to VHH-H14. ${ }^{11}$ Figure 1(B) shows the CD spectra of the VHH-R2-RR6 complex (line 1). The complex was formed by addition of the dye RR6 to a $1: 1$ molar ratio at $20^{\circ} \mathrm{C}$. The $\mathrm{CD}$ spectrum of VHH-R2 with RR6 is different from the CD spectrum of VHH-R2 alone.

The strong CD bands are probably due to stacking interactions of the aromatic side chains, that is, Trp52A in CDR2 of VHH-R2 is stacked against the naphtyl moiety of the azo-dye RR6 as shown in the X-ray structure of the complex. ${ }^{18}$ No signals have been observed in the CD spectrum of the hapten dye RR6 in PBS. Heating the VHH-R2-RR6 complex to $80^{\circ} \mathrm{C}$ did not change the CD spectra (line 2), even after incubation for $1 \mathrm{~h}$ at $80^{\circ} \mathrm{C}$. Subsequent cooling down of the complex does not alter the spectrum (line 3). This indicates that the complex retains its native conformation at this temperature. The complex of VHH-R2 with its antigen RR6 is more stable than VHH-R2 alone at high temperatures.

To obtain more insight into the stability of the complex at high temperatures, the VHH-R2 was heated to $80^{\circ} \mathrm{C}$ and after equilibration for $5 \mathrm{~min}$, the antigen RR6 was added to the unfolded VHH-R2. Figure 1(C) shows the CD spectrum of the unfolded VHH-R2 at $80^{\circ} \mathrm{C}$ (line 1). Subsequent addition of a 1:1 molar ratio of preheated RR6 to the antibody at $80^{\circ} \mathrm{C}$ resulted in the CD spectrum shown in Figure 1(C) (line 3). Surprisingly, this spectrum shows all the characteristics of the spectrum of the antibody-antigen complex formed at $20^{\circ} \mathrm{C}$ (line 2). This indicates that at $80^{\circ} \mathrm{C}$ the VHH-R2-RR6 complex adopts the same conformation as the complex formed at $20^{\circ} \mathrm{C}$.

Control experiments were performed regarding antigen specificity, antibody specificity and the effect of the copper in the dye on the CD spectra. To investigate whether the refolding was antigen specific, experiments were performed with VHH-R2 and the azo-dye RR1. The molecular structure of RR1 is different from RR6 and it does not contain copper like RR6 (Fig. 2).

ELISA experiments at room temperature showed that VHH-R2 could bind RR1, although with a $4 \times 10^{3}$-fold less affinity compared to antigen RR6 (data not shown). The CD spectrum of VHH-R2 after addition of the dye RR1 was different from the CD spectrum of VHH-R2 alone and also different from the CD spectrum of the VHH-R2-RR6 complex. 
A.

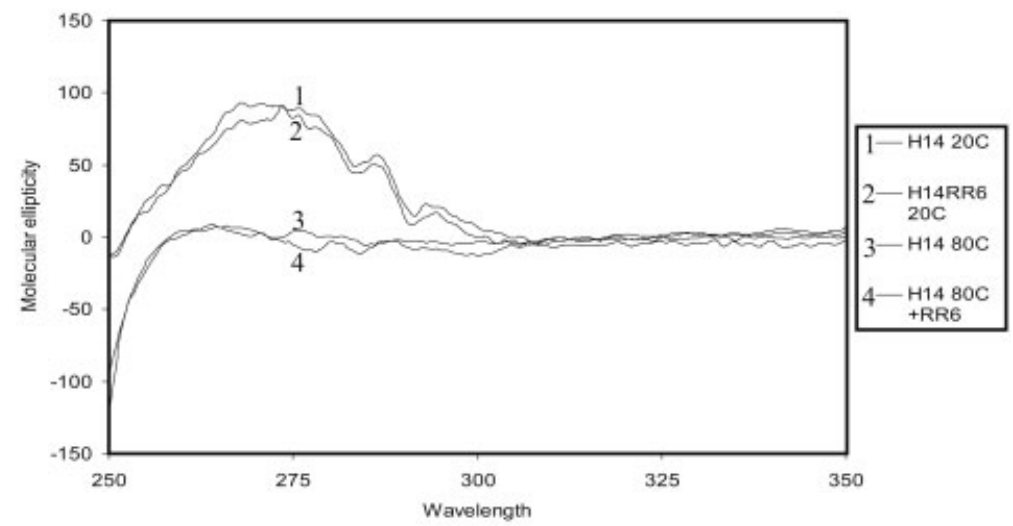

B.

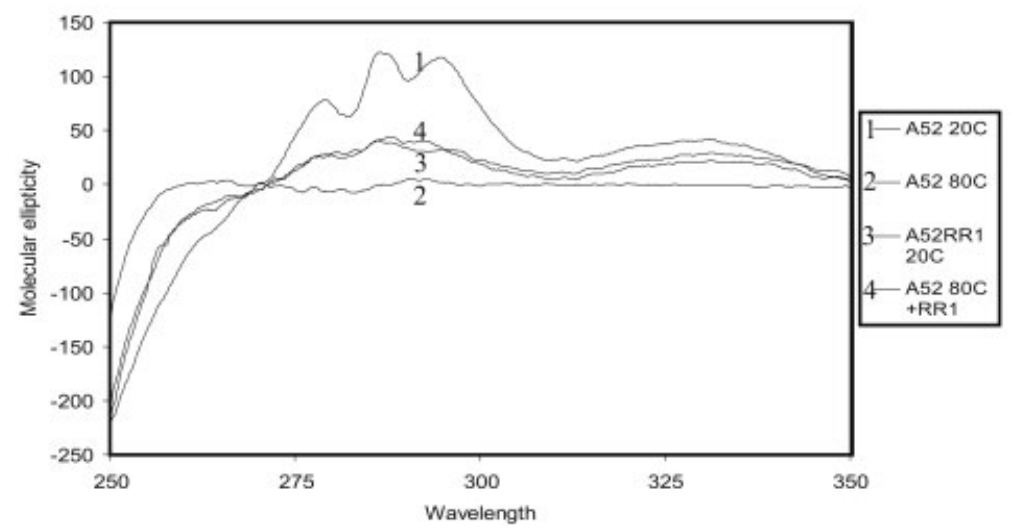

Fig. 3. (A) CD spectra in the near-UV area $\left(250-350 \mathrm{~nm}\right.$ ) of VHH-H14 at $20^{\circ} \mathrm{C}$ (line 1), with $\mathrm{RR} 6$ at $20^{\circ} \mathrm{C}$ (line 2), at $80^{\circ} \mathrm{C}$ (line 3), and with RR6 at $80^{\circ} \mathrm{C}$ (line 4). (B) CD spectra in the near- UV area (250-350 nm) of VHH-A52 at $20^{\circ} \mathrm{C}$ (line 1), at $80^{\circ} \mathrm{C}$ (line 2), of the VHH-A52:RR1 complex at $20^{\circ} \mathrm{C}$ (line 3), and of VHH-A52 at $80^{\circ} \mathrm{C}$ after addition of a 1:1 molar ratio of preheated RR1 (line 4).

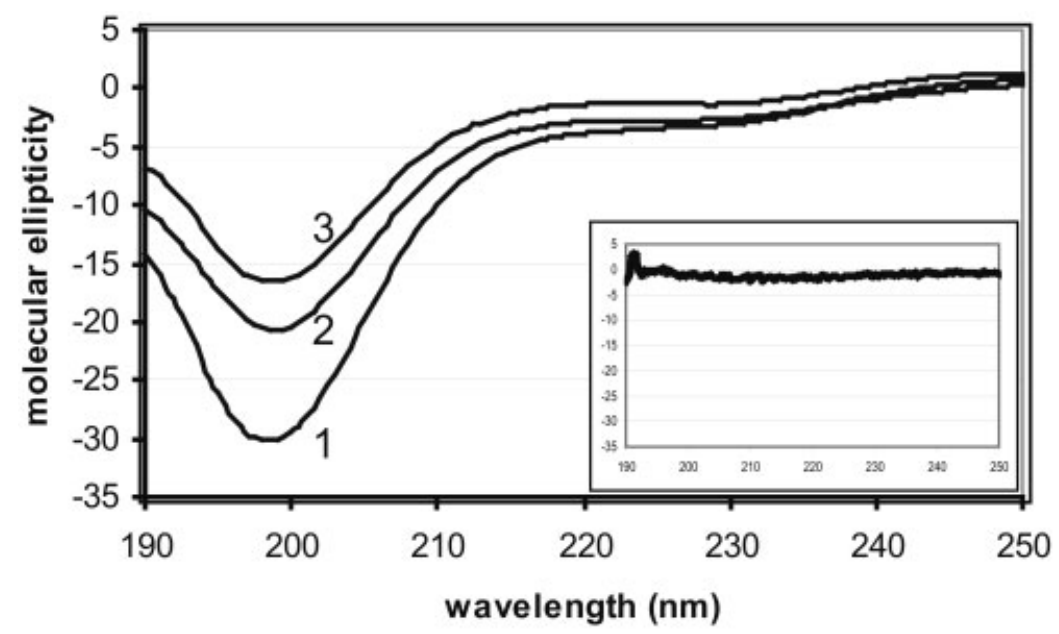

Fig. 4. CD spectra in the far-UV area (190-250 nm) of the CDR3 peptide without RR6 (line 1), after addition of a 1:1 molar ratio of RR6 (line 2), and after addition of a 1:3 molar ratio of RR6 (line 3). The inlay shows the control spectrum of RR6 alone (highest concentration). 
A

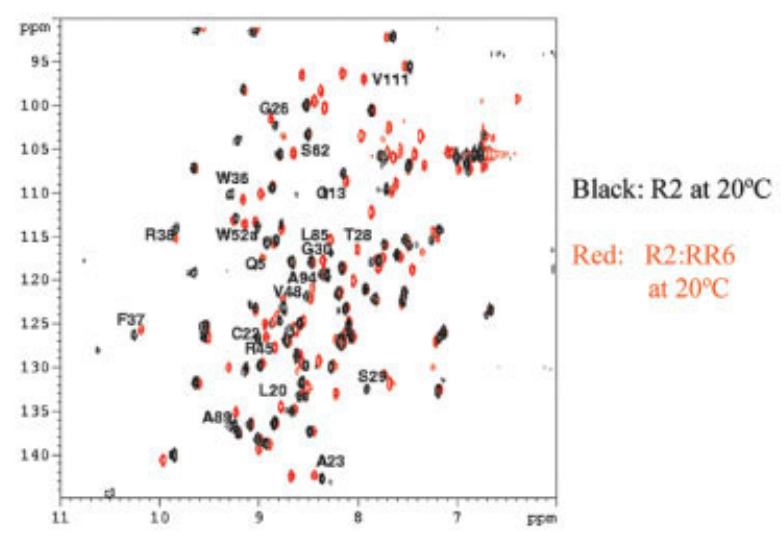

B

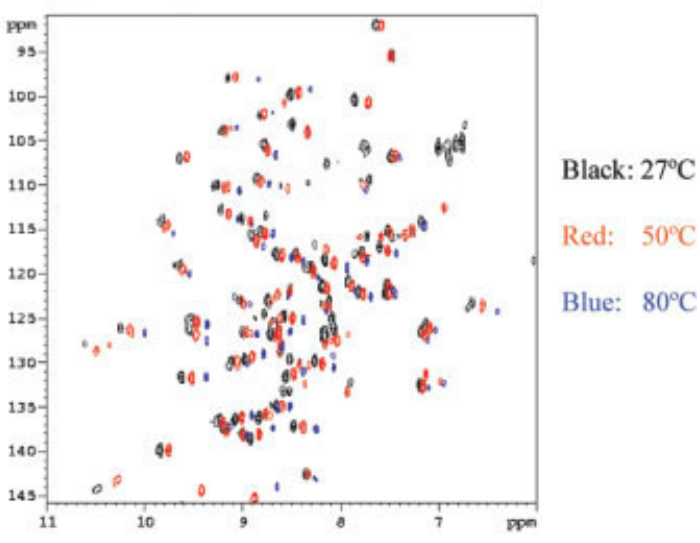

Fig. 5. (A) Superposition of the HSQC experiments of $0.1 \mathrm{mM} \mathrm{VHH-R2} \mathrm{(red)} \mathrm{and} \mathrm{VHH-R2:RR6} \mathrm{complex}$ (black) with the assignments of the shifted and broadened amide resonances. (B) Superposition of the HSQC experiments of the $\mathrm{VHH}-\mathrm{R} 2: \mathrm{RR} 6$ complex at three different temperatures: $27^{\circ} \mathrm{C}$ (black), $50^{\circ} \mathrm{C}$ (red), and $80^{\circ} \mathrm{C}$ (blue).

A

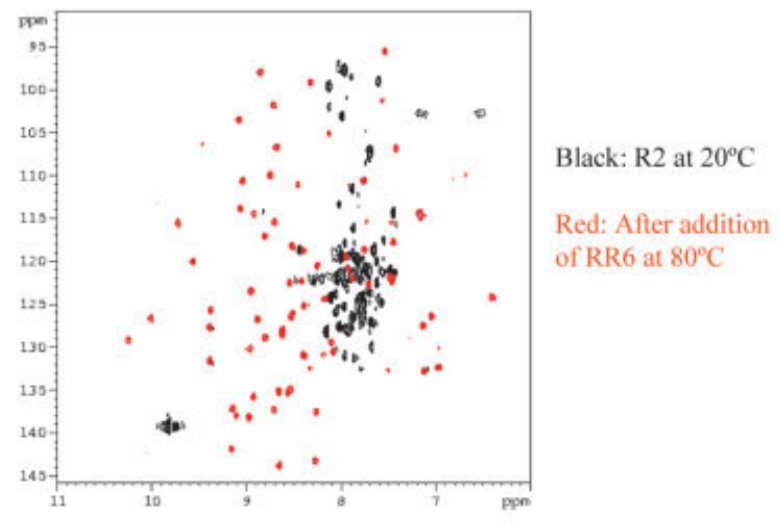

B

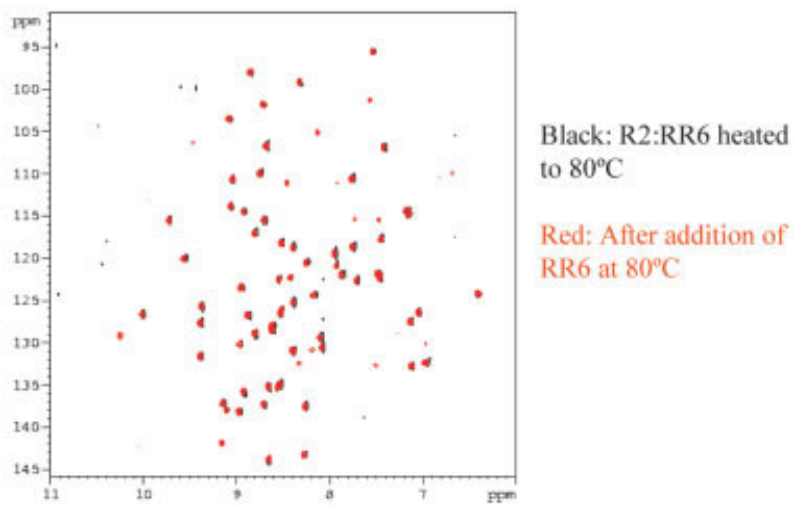

Fig. 6. (A) Superposition of the HSQC experiments of $0.1 \mathrm{mM}$ $\mathrm{VHH}-\mathrm{R} 2$ (black) at $80^{\circ} \mathrm{C}$ and after the addition of $0.1 \mathrm{mM}$ RR6 at the same temperature (red). (B) HSQC spectrum of the VHH-R2:RR6 complex heated to $80^{\circ} \mathrm{C}$ (black) superimposed with the spectrum of the complex prepared at $80^{\circ} \mathrm{C}$ (red).
This indicates that VHH-R2 is able to form a complex with RR1. However, addition of RR1 to the unfolded VHH-R2 at $80^{\circ} \mathrm{C}$ does not lead to the native complex of R2:RR1 (data not shown).

To ensure that the refolding of the VHH-R2 was not due to an aspecific interaction with RR6, the RR6 azo-dye was added to a different llama VHH fragment, VHH-H14 raised against hCG. Figure $3(\mathrm{~A})$ shows the CD spectrum of $\mathrm{H} 14$ alone at $20^{\circ} \mathrm{C}$ (line 1). Addition of RR6 did not alter the spectrum, indicating that the VHH-H14 does not interact with RR6 (line 2). Accordingly, at $80^{\circ} \mathrm{C}$ the $\mathrm{VHH}-\mathrm{H} 14$ is completely unfolded (line 3) and addition of the RR6 at $80^{\circ} \mathrm{C}$ did not alter the spectrum as well (line 4). To exclude that the copper alone has an effect on the CD spectrum of VHH-R2, CD experiments were performed with VHH-R2 in the presence of $\mathrm{CuCl}_{2}$ and $\mathrm{CuSO}_{4}$. Addition of $\mathrm{CuCl}_{2}$ or $\mathrm{CuSO}_{4}$ to $\mathrm{VHH}-\mathrm{R} 2$ at $20^{\circ} \mathrm{C}$ did not change the spectra of VHH-R2. This indicates that the copper is not responsible for the large bands in the CD spectrum of VHH-R2 (data not shown).

A different llama antibody fragment, VHH-A52 raised against the azo dye RR1, was used to determine whether the refolding at high temperatures was specific for VHH-R2 and RR6 or if it is a more generic process. The same set of experiments as with VHH-R2 was performed. Figure 3(B) shows the spectra of VHH-A52 (line 1) and the complex of VHH-A52:RR1 at $20^{\circ} \mathrm{C}$ (line 3). Heating the VHH-A52 to $80^{\circ} \mathrm{C}$ showed that the majority of the $\mathrm{VHH}-\mathrm{A} 52$ pool is in an unfolded conformation (line 2). Addition of RR1 to the unfolded VHH-A52 at $80^{\circ} \mathrm{C}$ led to complex formation and rendered a spectrum identical to the native complex spectrum at $20^{\circ} \mathrm{C}$ (line 4). This clearly indicates that VHH-A52 is capable of refolding upon addition of its antigen at $80^{\circ} \mathrm{C}$ just like VHH-R2 with its antigen RR6. Addition of RR 6 at $20^{\circ} \mathrm{C}$ and $80^{\circ} \mathrm{C}$ to $\mathrm{VHH}-\mathrm{A} 52$ also did not alter the spectra of VHH-A52 (data not shown). These observations indicate that refolding at $80^{\circ} \mathrm{C}$ upon addition 


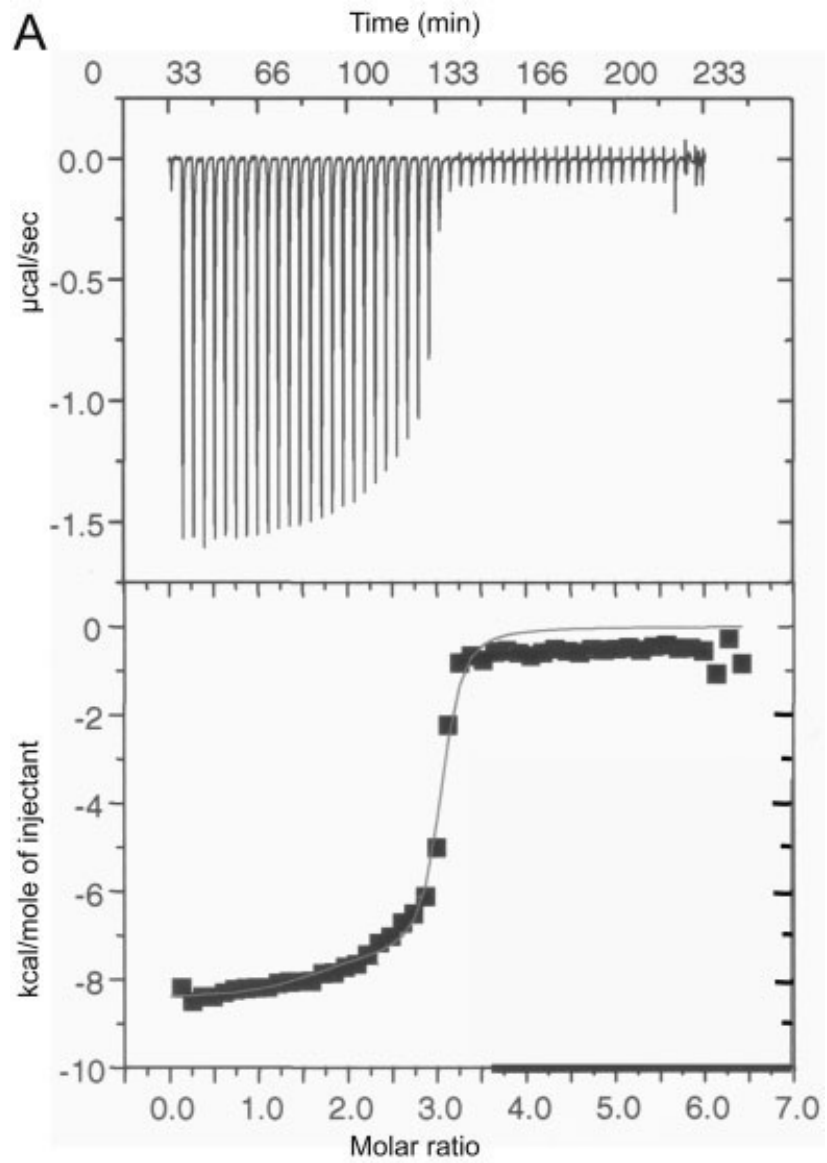

of the antigen was not specific for VHH-R2, but could be generic for the llama antibody fragments.

\section{Peptide Binding}

To investigate whether an interaction between denatured VHH-R2 and RR6 is possible, a peptide was synthesized comprising the CDR3 binding loop of VHH-R2. Figure 4 shows the CD spectrum of the peptide (line 1). The spectrum of the peptide indicates that the CDR3 peptide is largely in an unordered random coil formation. Addition of antigen RR6 to the peptide renders a spectrum as shown in Figure 4. This shows that there is an interaction between RR6 and the peptide. The spectra with dye shows a shift in the 190-210 $\mathrm{nm}$ range, which indicates that the antigen RR6 induced a more structured conformation of the peptide. All spectra shown are corrected for the spectrum of the buffer or buffer with the corresponding concentration of RR6. Therefore, the shift of the curve cannot be an effect of $\mathrm{pH}$. These results indicate that the antigen RR6 is able to interact with the unordered CDR3 peptide.

\section{NMR}

To obtain more details on the structure of VHH-R2 upon binding of the dye RR6, NMR experiments were performed. HSQC spectra provide information on the chemical shift of the amide resonances in the backbone of R2. A well-dispersed amide crosspeak pattern is representative for a native protein. Figure 5(A) shows the superimposed HSQC spectra of free ${ }^{15} \mathrm{~N}-\mathrm{VHH}-\mathrm{R} 2$ (red) and the ${ }^{15} \mathrm{~N}-\mathrm{VHH}-$ R2:RR6 complex (black) at $27^{\circ} \mathrm{C}$. Both spectra clearly show that ${ }^{15} \mathrm{~N}-\mathrm{VHH}-\mathrm{R} 2$ and its complex sample is folded. Moreover, a few crosspeaks in the spectrum of the ${ }^{15} \mathrm{~N}-\mathrm{VHH}-\mathrm{R} 2$ : RR6 complex were shifted, and some of them were even undetectable. This indicates that these resonances probably correspond to the residues in close proximity to RR6 or to residues which undergo long-range conformational changes upon binding of RR6. The reason that some of the crosspeaks are undetectable is due to line-broadening induced by the interaction of the resonating nuclei with the unpaired electron of the paramagnetic copper center in RR6. The relaxation of the protons in close proximity to the paramagnetic center is fast and leads to moderately/ largely broadened signals beyond detection limit.

The chemical shift assignments for most of the perturbed residues have been obtained and are assigned in Figure 5(A). The broadened crosspeaks in the spectrum are L20, T28, S29, G30, R45, W52a, S62, A94, and V111. In the X-ray structure W52a is in stacking contact with the naphtyl moiety of RR6, and is therefore undetectable in the HSQC experiment of the complex. ${ }^{18}$ Unfortunately, the NMR resonance assignments of the coordinating ligand of the copper, H31a and H31c, are still missing. A few

Fig. 7. (A) Binding isotherm of the titration of RR6 to VHH-R2 at $20^{\circ} \mathrm{C}$. Top panels are the raw ITC data. Lower panels are the integrated calorimetric heats. (B) Binding isotherm of the titration of RR6 to VHH-R2 at $80^{\circ} \mathrm{C}$. Top panels are the raw ITC data. Lower panels are the integrated calorimetric heats. 
TABLE I. ITC Data

\begin{tabular}{|c|c|c|c|c|c|c|c|c|}
\hline & N1 & $\begin{array}{c}\mathrm{Ka} 1 \\
\left(M^{-1}\right)\end{array}$ & $\Delta \mathrm{H} 1(\mathrm{~J} / \mathrm{mol})$ & $\Delta \mathrm{S} 1(\mathrm{~J} / \mathrm{mol} / \mathrm{k})$ & N2 & $\begin{array}{c}\mathrm{Ka} 2 \\
\left(M^{-1}\right)\end{array}$ & $\Delta \mathrm{H} 2(\mathrm{~J} / \mathrm{mol})$ & $\Delta \mathrm{S} 2(\mathrm{~J} / \mathrm{mol} / \mathrm{k})$ \\
\hline $\mathrm{RR} 6$ to $\mathrm{R} 2$ at $20^{\circ} \mathrm{C}$ & 1.4 & $3.4 \times 10^{6}$ & -7400 & 4.6 & 1.6 & $4.6 \times 10^{7}$ & -8446 & 6.3 \\
\hline $\mathrm{RR} 6$ to $\mathrm{R} 2$ at $80^{\circ} \mathrm{C}$ & 1.4 & $1.9 \times 10^{6}$ & $-3.9 \times 10^{4}$ & -83 & $6.1^{*}$ & $350.2^{*}$ & $1.7 \times 10^{6 *}$ & $503^{*}$ \\
\hline
\end{tabular}

The calorimetric data corresponding to the RR6 titrations at $20^{\circ} \mathrm{C}$ and $80^{\circ} \mathrm{C}$ fitted using a model assuming two binding events. The asterix indicates that the accuracy of these results are poor.

other residues in CDR1 close to the histidines coordinating the copper, that is, T28, S29, and G30 were also severely broadened in the complex. The rest of the undetectable residues with the exception of L20 were either part of or are close to the CDRs.

The ${ }^{15} \mathrm{~N}-\mathrm{VHH}-\mathrm{R} 2: \mathrm{RR} 6$ complex was heated from $27^{\circ} \mathrm{C}$ to $80^{\circ} \mathrm{C}$ [Fig. 5(B)]. The dispersion of the amide resonances remained roughly the same up to $80^{\circ} \mathrm{C}$ except for a small shift of the peaks not representative of large conformational changes.

However, the intensity of the crosspeaks dropped substantially at higher temperatures due to line broadening, which could be indicative of aggregation. In contrast, when free ${ }^{15} \mathrm{~N}-\mathrm{VHH}-\mathrm{R} 2$ is heated to $80^{\circ} \mathrm{C}$ a large shift of the amide resonances to random coil chemical shift values has been observed [Fig. 6(A)]. This suggests that the majority of the population of the ${ }^{15} \mathrm{~N}-\mathrm{VHH}-\mathrm{R} 2$ molecules is unfolded at $80^{\circ} \mathrm{C}$. Addition of an equivalent molar amount of RR6 to denatured ${ }^{15} \mathrm{~N}-\mathrm{VHH}-\mathrm{R} 2$ at $80^{\circ} \mathrm{C}$ rendered a dramatically altered spectrum [Fig. 6(A)]. Remarkably, this spectrum appeared to be identical to that from the ${ }^{15} \mathrm{~N}-\mathrm{VHH}-\mathrm{R} 2$ :RR6 complex prepared at $27^{\circ} \mathrm{C}$ and subsequently heated to $80^{\circ} \mathrm{C}$ [Fig. 6(B)]. This finding gives direct evidence for the refolding of nonnative ${ }^{15} \mathrm{~N}-\mathrm{VHH}-\mathrm{R} 2$ at $80^{\circ} \mathrm{C}$ upon binding of its antigen RR6. The NMR experiments were performed at $\mathrm{pH} 4.6$ to minimize the exchange of amide protons. Deviations because of differences in $\mathrm{pH}$ between the $\mathrm{CD}$ and NMR experiments were checked by repeating the CD experiments at $\mathrm{pH} 4.6$; no differences were observed. The NMR data confirms the CD data and gives direct evidence for the hypothesis of "induced refolding."

\section{Isothermal Titration Calorimetry}

To understand the thermodynamics involved in the refolding process isothermal titration calorimetry (ITC) was performed. Small amounts of RR6 were titrated to VHH-R2 at $20^{\circ} \mathrm{C}$ and $80^{\circ} \mathrm{C}$. Figure $7(\mathrm{~A})$ shows a typical binding isotherm of RR6 to VHH-R2 at $20^{\circ} \mathrm{C}$.

The measured heat change for each injection is negative up to a molar ratio of 3 , after which it remains constant up to 7 equivalents of RR6. The exothermic signals of -8 $\mathrm{kcal} / \mathrm{mol}$ at molar ratios up to 3 represent the heat change of complex formation of RR6 to VHH-R2. The enthalpic signals at molar ratios between 3 and 7 represent the heat of dilution of the RR6 and are observed in a control titration, wherein RR6 was titrated to PBS buffer only. Figure $7(\mathrm{~B})$ shows the binding isotherm at $80^{\circ} \mathrm{C}$. The measured heat change for each injection is negative up to a molar ratio of 1.5, after which it remains constant up to 7.0 equivalents of RR6. The exothermic signals of $-39 \mathrm{kcal} /$

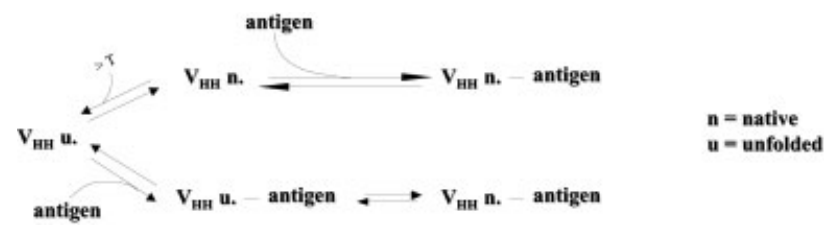

Fig. 8. A reaction scheme of a native and unfolded $\mathrm{VHH}$ and its antigen towards complex formation with two suggestions for the role of addition of antigen in induced refolding at high temperature.

mol represent the complex formation of RR6 to VHH-R2 and the refolding of VHH-R2.

Values for the enthalpy change and the number of binding sites $(N)$ correspond to the intercepts and midpoints, respectively, of the titration curves and can be obtained directly from the ITC data. Both curves were fitted with a model assuming two binding events, because two RR6 molecules were shown to bind to VHH-R2 ${ }^{18}$ (see Table I).

The binding isotherms of the titration of RR6 to VHH-R2 at $20^{\circ} \mathrm{C}$ and $80^{\circ} \mathrm{C}$ shows that titration of RR6 is exothermic at both temperatures. The observed enthalpy change indicates that refolding takes place at $80^{\circ} \mathrm{C}$ and confirms the $\mathrm{CD}$ data. At $20^{\circ} \mathrm{C}$, the thermodynamic parameters of the binding of the first and the second RR6 molecule are similar. At $80^{\circ} \mathrm{C}$, the thermodynamic parameters of the binding of the first RR6 molecule are comparable to the parameters at $20^{\circ} \mathrm{C}$, although the association constant is slightly lower.

However, the thermodynamic parameters of association of the second RR6 molecule are drastically different, suggesting that at $80^{\circ} \mathrm{C}$ the second RR6 molecule is not able to bind efficiently. The data obtained from the crystal structure of the complex show two binding sites for RR6; however, the RR6 monomer was the authentic hapten and our model is based on binding of a monomer RR6 by VHH-R2. The large enthalpic change of $-35 \mathrm{kcal} / \mathrm{mol}$ at $80^{\circ} \mathrm{C}$ can be explained because not only binding and complex formation, like at $20^{\circ} \mathrm{C}$, but also refolding occurs, as was shown in the $\mathrm{CD}$ experiments. The ITC data confirm the CD data, and show that the thermodynamic parameters of binding at $80^{\circ} \mathrm{C}$ is remarkably comparable to binding at $20^{\circ} \mathrm{C}$.

\section{CONCLUSION}

We have shown by CD spectroscopy and NMR that unfolded VHH-R2 was able to form a native complex after addition of its antigen RR6 at high temperature, indicative of refolding of $\mathrm{VHH}-\mathrm{R} 2$ at $80^{\circ} \mathrm{C}$. The thermodynamic data obtained with ITC also show a favorable RR6 binding 

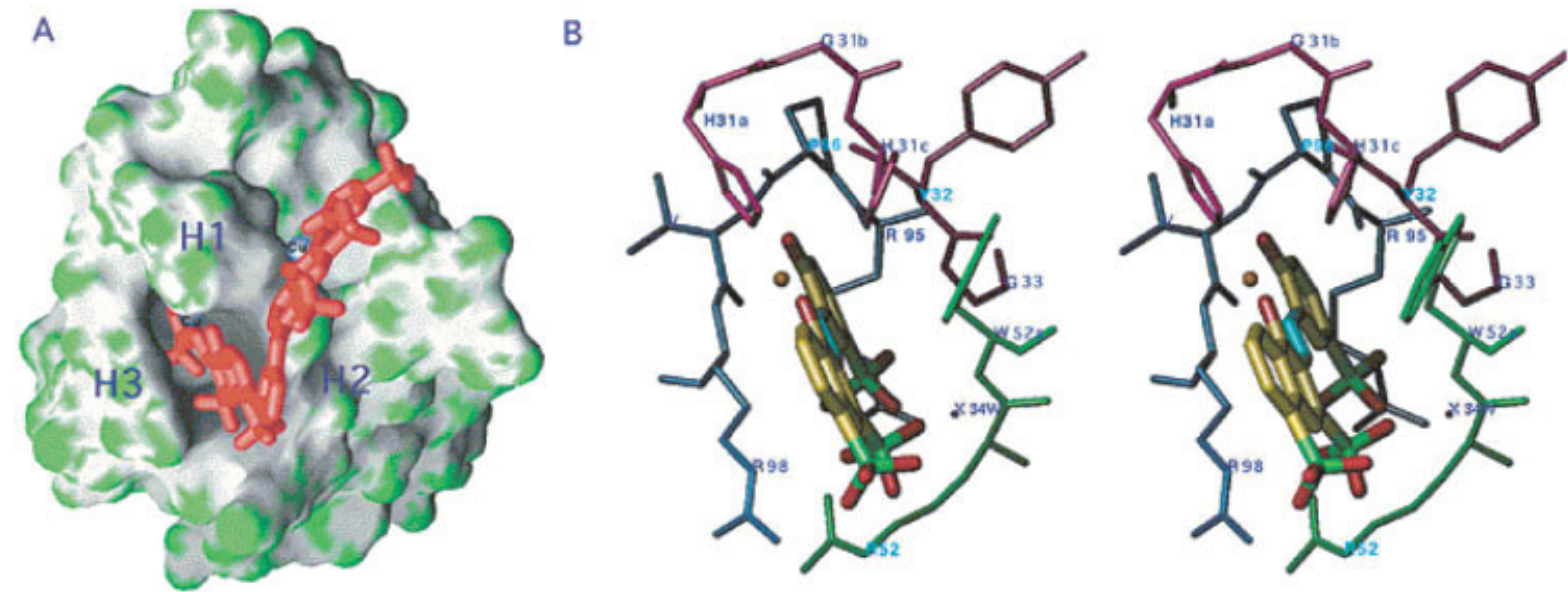

Fig. 9. The combining site of the anti-RR6-VHH and the RR6 inside, viewed from the top. (A) Water-accessible surface of the VHH displaying the cavities, with the bound RR6 molecule (red) in ball-and-stick representation, with the copper ions as silvery blue spheres. The green color identifies the protruding surfaces, and the gray color identifies the reentring surfaces (GRASP representation, 32). The localization of the three CDRs has been indicated by H1, H2, and $\mathrm{H} 3$. (B) Stereoview of the P1 and N1 groups of the P1 + N1 groups of the RR6 molecule in complex with the three CDRs of the anti-RR6-VHH fragment. The CDRs 1-3 are red, green, and blue, respectively. (Reproduced with permission from Biochem. 2000;39:1217-1222. Copyright ${ }^{\odot} 2000$ Am Chem Soc).

enthalpy at $80^{\circ} \mathrm{C}$, indicative of refolding of VHH-R2 at $80^{\circ} \mathrm{C}$. Although this is the most likely explanation, we cannot determine the extent to which the enthalpy change is due to protein folding or RR6 binding. Figure 8 shows a scheme of the possible steps in the binding process of a VHH to its antigen at $80^{\circ} \mathrm{C}$.

A possible pathway for complex formation at these high temperatures is that the small fraction of native VHH-R2 at $80^{\circ} \mathrm{C}$ is able to form a complex with the dye RR6. Consequently, the equilibrium will set, according to the law of mass action, leading to a new equilibrium with the majority of the VHH-R2 in complex with RR6. Another pathway for complex formation could be that the antigen first binds to unfolded VHH-R2, which subsequently induces the refolding process. Figure 4 in this article shows that an interaction between the unordered CDR3 peptide and the dye RR6 is possible. This can be explained in more detail after careful inspection of the X-ray structure. The X-ray data showed a dimer of RR6 bound to the VHH; however, only one RR6 molecule of the dimer is buried in a cavity at the surface of the VHH. ${ }^{18}$ This first half of the dimer is buried for $60 \%$ in the $\mathrm{VHH}$, embedded between the three CDRs. Binding of a RR6 molecule to CDR3 increases the probability that the RR6 molecule binds to a second CDR, resulting in formation of the cavity as is present in the native VHH-R2.

RR6 was shown to induce structural changes in the unstructured CDR3 peptide. These structural changes in unfolded VHH-R2 could increase the probability of reformation of the loops of the CDRs. This could be the folding nuclei that allows long-range interacting residues to come in more proximity of each other and refold to the correct native conformation, thereby trapping the unfolded VHH-R2 into a stable conformation, the VHH-R2:RR6 complex. The formation of a folding nuclei has been described before for folding of immunoglobulin-like proteins as the nucleation-condenzation mechanism. ${ }^{19-21}$ In this case, the antigen RR6 would act like a small chaperone molecule, which acts by increasing the probability of unfolded proteins to form a stable structure. ${ }^{22-24}$ However, the difference is that small chaperone molecules often bind to regions that are buried in the native protein, but exposed in the unfolded protein, whereas RR6 binds to the CDRs that are also exposed in the folded VHHs.

It is most likely that both pathways exist next to each other. Irrespective of the condition, there will always be an equilibrium between folded and unfolded VHH-R2; thus folded VHH-R2 is present. This amount of folded VHH-R2 will form a complex upon addition of the antigen RR6. Next to this pathway, we have indications that another pathway is possible, via interaction of the unfolded VHH-R2 with the antigen RR6.

The main difference between these two pathways is whether RR6 binds to the unfolded VHH-R2 or to the native VHH-R2 at $80^{\circ} \mathrm{C}$. In this perspective, it was interesting to see that VHH-R2 was able to bind azo-dye RR1 at $20^{\circ} \mathrm{C}$, albeit with a lower affinity than azo-dye RR6. However, addition of RR1 to the unfolded VHH-R2 at $80^{\circ} \mathrm{C}$ did not induce measurable refolding of VHH-R2. The interaction of RR1 with VHH-R2 is probably not sufficient to induce refolding. Comparing the molecular structures of RR1 and RR6, there are some remarkable resemblances: the aromatic rings, the three sulphonate groups, and the sulfonyl-phenol group. Inspection of the contacts of VHH-R2 with RR6 shows that CDR1 is in contact with RR6 via the copper molecule; CDR2 is in contact with the three sulphonate groups of RR6 and CDR3 with the $p$-sulfonyl-phenol group of RR6 (Fig. 9). RR1 does not contain a copper, the sulphonate groups of RR1 are differently orientated compared to RR6, and thus it is likely that the contact of RR1 to VHH-R2 is with the identical $p$-sulfonyl-phenol group of RR1 to CDR3 of VHH-R2. The reason why RR1 is not able to induce refolding by long range interactions could be that stabilization of the CDR loop(s) followed by refolding is not 
efficient with the antigen RR1 or that the time necessary for association is too short to form a stable complex due to the low affinity of VHH-R2 for RR1.

VHH-A52, a VHH selected against and binding specifically to the azo-dye RR1, did show refolding after addition of $\mathrm{RR} 1$ at $80^{\circ} \mathrm{C}$. In this case, the interaction of $\mathrm{RR} 1$ with VHH-A52 is sufficient to induce refolding. RR1 is not only able to bind to VHH-A52, but is also capable of inducing long range interactions in VHH-A52 necessary to refold VHH-A52 to its correct native conformation. Spinelli reported the structure of VHH-A52 with RR1. ${ }^{25}$ Where VHH-R2 forms a cavity with its three CDRs and binds RR6 in a central combining site, VHH-A52 binds RR1 on a lateral combining site with CDR2, CDR3, and a framework residue. Thus, although the mechanism for binding their antigen is different between VHH-A52 and VHH-R2, both VHHs are able to refold upon addition of their antigen at $80^{\circ} \mathrm{C}$. We concluded that refolding at $80^{\circ} \mathrm{C}$ upon addition of the antigen is not specific for the $\mathrm{VHH}-\mathrm{R} 2$, but is generic for the llama antibody fragments against organic haptens. However, high-affinity binding of $\mathrm{VHH}$ to its antigen is a necessity to enable long-range interactions to occur or ensures the association time necessary for stable complex formation and shift the equilibrium.

The binding of camelid single domain antibody fragments under extreme conditions increases the possibilities to use these simple binding molecules in a wide range of applications. They are not only suitable for applications where a relatively short rise in temperature is necessary like with sterilization, but they may also be used in application at higher temperature or under different denaturating conditions. Because of the single domain "induced refolding" is a unique characteristic of llama antibody fragments in general.

This article shows that the increased stability reported in many articles concerning single-domain antibodies derived from camelids might be contributed in large part by their reversible folding and capability of binding to their antigen under extreme conditions. This was shown by the fact that the unfolded $\mathrm{VHH}$ was still functional, that is, able to efficiently bind to their antigen to a great extend in a relatively short time span.

\section{ACKNOWLEDGMENTS}

The authors acknowledge Ingrid Schaffers and Ewout van Velzen for practical assistance, and Maarten Egmond and Ruud Cox for fruitful discussions and synthesis of the peptide. The AFMB research group is greatly acknowledged for technical assistance.

\section{REFERENCES}

1. Neet KE, Koshland DE. Conversion of serine at the active site of subtilisin to cysteine. A "chemical mutation." Proc Natl Acad Sci USA 1966;56:1606-1611.

2. Webster DM, Henry AH, Rees AR. Antibody-antigen interactions. Curr Opin Struct Biol 1994; 4:123-129.

3. Burley SK. The more things change, the more they stay the same. Nat Struct Biol 1994;1:207-208.

4. McEwan IJ, Dahlman-Wright K, Ford J, Wright, APH. Functional interaction of the c-Myc transactivation domain with the TATA binding protein: evidence for an induced fit model of transactivation domain folding. Biochemistry 1996;35:9584-9593.
5. Hamers-Casterman C, Atarhouch T, Muyldermans S, Robinson G, Hamers C, Songa EB, Bendahman N, Hamers R. Naturally occurring antibodies devoid of light chains. Nature 1993;363:446448.

6. Van der Linden RHJ, Frenken LGJ, de Geus B, Harmsen MM, Ruuls RC, Stok W, de Ron L, Wilson S, Davis P, Verrips CT. Comparison of physical chemical properties of llama V-HH antibody fragments and mouse monoclonal antibodies. Biochim Biophys Acta 1999;1431:37-46.

7. Muyldermans S, Cambillau C, Wyns L. Recognition of antigens by single-domain antibody fragments: the superfluous luxury of paired domains. Trends Biosci 2001;26:230-235.

8. Frenken LGJ, Hessing JGM, Van den Hondel CAMJ, Verrips CT. Recent advances in the large-scale production of antibody fragments using lower eukaryotic microorganisms. Res Immunol 1998;149:589-599.

9. Frenken LGJ, van der Linden RHJ, Hermans PWJJ, Bos JW, Ruuls RC, de Geus B, Verrips CT. Isolation of antigen specific Llama V-HH antibody fragments and their high level secretion by Saccharomyces cerevisiae. J Biotech 2000;78:11-21.

10. Thomassen YE, Meijer W, Sierkstra L, Verrips CT. Large-scale production of VHH antibody fragments by Saccharomyces cerevisiae. Enzyme Microb Technol 2002;30:273-278.

11. Pérez JMJ, Renisio JG, Prompers JJ, van Platerink CJ, Cambillau C, Darbon H, Frenken LGJ. Thermal unfolding of a Llama antibody fragment: a two-state reversible process. Biochemistry 2001;40:74-83.

12. Van der Linden R, de Geus B, Stok W, Bos W, van Wassenaar D, Verrips T, Frenken L. Induction of immune responses and molecular cloning of the heavy chain antibody repertoire of Lama glama. J Immunol Methods 2000;240:185-195.

13. Verheesen P, ten Haaft MR, Lindner N, Verrips CT, de Haard JJ. Beneficial properties of single-domain antibody fragments for application in immunoaffinity purification and immuno-perfusion chromatography. Biochim Biophys Acta 2003;1624:21-28.

14. Zhu H, Klemic JF, Chang S, Bertone P, Casamayor A, Klemic KG, Smith D, Gerstein M, Reed MA, Snyder M. Analysis of yeast protein kinases using protein chips. Nat Genet 2000;26:283-289.

15. Spinelli S, Frenken L, Bourgeois D, de Ron L, Bos W, Verrips T, Anguille C, Cambillau C, Tegoni M. The crystal structure of a llama heavy chain domain. Nat Struct Biol 1996;3:752-757.

16. Mori S, Abeygunawardana C, Johnson MO, van Zijl PCM. Improved sensitivity of HSQC spectra of exchanging protons at short interscan delays using a new fast HSQC (FHSQC) detection scheme that avoids water saturation. J Magn Reson 1995;B108:9498

17. Marion D, Ikura M, Tschudin R, Bax A. Rapid recording of 2D NMR spectra without phase cycling: application to the study of hydrogen exchange in proteins. J Magn Reson 1989;85:393-399.

18. Spinelli S, Frenken LG, Hermans P, Verrips T, Brown K, Tegoni M, Cambillau C. Camelid heavy-chain variable domains provide efficient combining sites to haptens. Biochemistry 2000;39:12171222.

19. Fersht AM. Optimization of rates of protein folding: the nucleationcondensation mechanism and its implications. Proc Natl Acad Sci USA 1995;92:10869-10873.

20. Vendruscolo M, Paci E, Dobson CM, Karplus M. Three key residues form a critical contact network in a transition state for protein folding. Nature 2001;409:641-646.

21. Wright CF, Christodoulou J, Dobson CM, Clarke J. The importance of loop length in the folding of an immunoglobulin domain. Protein Eng Des Sel 2004. Advance Access published online on June 18, 2004.

22. Hendrick JP, Hartl FU. The role of molecular chaperones in protein folding. FASEB J 1995;9:1559-1569.

23. Ellis RJ, Hartl FU. Principles of protein folding in the cellular environment. Curr Opin Struct Biol 1999;9:102-110.

24. Torok Z, Goloubinoff P, Horvath I, Tsvetkova NM, Glatz A, Balogh G, Varvasovszki V, Los DA, Vierling E, Crowe JH, Vigh L. Synechocystis HSP17 is an amphitropic protein that stabilizes heat-stressed membranes and binds denatured proteins subsequent chaperone-mediated refolding. Proc Natl Acad Sci USA 2001;98:3098-3103.

25. Spinelli S, Tegoni M, Frenken L, van Vliet C, Cambillau C. Latera recognition of a dye hapten by a llama VHH domain. J Mol Biol 2001;311:123-129. 